\begin{tabular}{|c|c|}
\hline Title & Eddy Current A nalysis of Litz Wire U sing Homogenization-Based FEM in Conjunction With Integral Equation \\
\hline Author(s) & Hiruma, Shingo; Otomo, Y oshitsugu; Igarashi, Hajime \\
\hline Citation & $\begin{array}{l}\text { IEEE Transactions on Magnetics, 54(3), } 7001404 \\
\text { https://doi.org/10.1109/ MA G.2017.2772335 }\end{array}$ \\
\hline Issue Date & 201803 \\
\hline Doc URL & http:/hdl.handle.net/2115/68966 \\
\hline Rights & $\begin{array}{l}\text { (C) } 2017 \text { IEEE. Personal use of this material is permitted. Permission from IEEE must be obtained for all other uses, in } \\
\text { any current or future media, including reprinting/republishing this material for advertising or promotional purposes, } \\
\text { creating new collective works, for resale or redistribution to servers or lists, or reuse of any copy righted component of } \\
\text { this work in other works. }\end{array}$ \\
\hline Tyре & article (author version) \\
\hline File Information & manuscript.pdf \\
\hline
\end{tabular}

Instructions for use 


\title{
Eddy Current Analysis of Litz Wire Using Homogenization-based FEM in Conjunction with Integral Equation
}

\author{
Shingo Hiruma ${ }^{1}$, Yoshitsugu Otomo ${ }^{1}$, Hajime Igarashi ${ }^{1}$, Member, IEEE \\ ${ }^{1}$ Graduate School of Information Science and Technology, Hokkaido University, 060-0814 Sapporo, Japan
}

\begin{abstract}
A new method is introduced to evaluate the macroscopic permeability of a litz wire which is composed of stranded conductors. In this method, an integral equation is solved for the complex magnetization in the litz wire generated due to the proximity effect. The macroscopic permeability computed from the magnetization is used in the homogenization-based finite element analysis of eddy currents in a litz-wire coil. It is shown that the wire twist has little effect on the complex permeability.
\end{abstract}

\section{Index Terms - Homogenization method, integral equation, litz wire, macroscopic permeability, Ollendorff formula}

\section{INTRODUCTION}

$\mathbf{I}_{\mathrm{s} m}^{\mathrm{N}}$ $\mathrm{N}$ electric machines and devices, materials which involve small structures such as litz wire, steel sheet, dust core, and so on, are widely used to reduce the eddy current loss. In recent years, analysis of eddy current losses in these materials has been required because of increase in speed of the switching frequency of power electronics devices. When analyzing those materials by the space-discretization method such as the finite element method (FEM), the resultant equation can be extremely large because they have to be discretized into huge number of elements that are smaller than the skin depth.

The homogenization-based FEM has been shown effective to such analysis [1-7]. In [5], complex permeability has been introduced for the analysis of multi-turn coils. The macroscopic permeability of a coil region is obtained by substituting the complex permeability of a round conductor to the extended Ollendorff formula. The homogenization method allows us to avoid discretization of the components in fine-structured material into small finite elements because it is possible to treat it as uniform material. However, in the homogenization-based FEM, the stranded structure of a litz wire has been ignored.

In [8], to consider the stranded structure, an integral equation method to analyze the eddy current losses in the stranded wire has been proposed by the authors. In this method, the integral equation is solved for the magnetization in the wire generated by the proximity effect. In this paper, we show that the macroscopic permeability of the litz wire can be evaluated using the integral equation method. To do so, uniform magnetic field is imposed to a litz wire, and the integral equation is solved to evaluate the eddy currents in the litz wire. Then, the macroscopic permeability is derived from the computed magnetic field for the following FE analysis. We discuss validation of the proposed method by comparing the results obtained by the proposed method and conventional FEM for a

Manuscript received April 1, 2015; revised May 15, 2015 and June 1, 2015; accepted July 1, 2015. Date of publication July 10, 2015; date of current version July 31, 2015. Corresponding author: S. Hiruma (e-mail: hiruma@em.ist.hokudai.ac.jp). If some authors contributed equally, write here, "Y. Otomo and H. Igarashi contributed equally." IEEE TRANSACTIONS ON MAGNETICS discourages courtesy authorship; please use the Acknowledgment section to thank your colleagues for routine contributions. Digital Object Identifier (inserted by IEEE). litz wire. Using the proposed method, we evaluate the dependence of the macroscopic permeability on the pitch of the litz wire. Moreover, the numerical results are compared with measured values.

\section{COMPLEX PERMEABILITY OF STRANDED WIRES}

\section{A. Complex permeability of a round wire}

When a wire is immersed in time-harmonic magnetic field which is perpendicular to the wire axis, anti-parallel eddy currents are induced along the wire. Because the eddy currents generate the diamagnetic field, the effective permeability of the wire becomes lower than its specific permeability. Moreover, the eddy currents give rise to Joule losses. These effects can be expressed by the complex permeability. The relative complex permeability of a round wire is given by [5]

$\dot{\mu}_{r}=\mu_{r} \frac{J_{1}(z)}{z J_{l}^{\prime}(z)}$

where $z=(1-j) / \delta, \delta$ denotes the skin depth, $J_{1}(z)$ represents the first-order Bessel function, and $\mu_{r}$ is the relative permeability of the wire. Using this permeability, we can consider the eddy currents losses due to the proximity effect without fine discretization of the wire cross section.

\section{B. Periodic integral equation}

The anti-parallel eddy currents along stranded wires induced by a uniform magnetic field can be obtained by the integral equation method in which a wire is modeled as one-dimensional curve [8]. Assuming that wires have periodic structure, the electromagnetic field around the wire can be obtained by considering a unit cell under the periodic boundary condition.

The basic idea of the integral equation method is that the antiparallel eddy currents in the wire due to the proximity effect generate a dipole field. The source of the dipole field can be regarded as a magnetization in the wire. Therefore, if the distribution of the magnetization is obtained, the anti-parallel eddy currents in the wire can be determined.

In the following, we derive the integral equation for selfconsistently determining the distribution of the magnetization in stranded wires. Let us consider the complex magnetization 
$\boldsymbol{M}$ perpendicular to the wire axis generated by the anti-parallel eddy currents. Considering the eddy currents, the magnetization is expressed in terms of the magnetic field $\boldsymbol{H}$ and $\dot{\mu}$ as

$$
\boldsymbol{M}=\left(\dot{\mu}_{\boldsymbol{r}}-1\right) \boldsymbol{H}
$$

The magnetic field $\boldsymbol{H}$ is composed of the components due to the complex magnetization vector $\boldsymbol{M}$ distributed along the wire and the uniform magnetic field $\boldsymbol{H}_{0}$. Then we obtain the following integral equation from (2) for $\boldsymbol{M}$ [8]:

$$
\begin{aligned}
& \frac{\boldsymbol{M}(\boldsymbol{x})}{\dot{\mu}_{r}-1}=\boldsymbol{\tau} \times\left[\left(\int_{\Omega_{c}} G \boldsymbol{M d v ^ { \prime }}+\boldsymbol{H}_{0}(\boldsymbol{x})\right) \times \boldsymbol{\tau}\right] \\
& G \boldsymbol{M}=-\frac{1}{4 \pi}\left[\frac{\boldsymbol{M}\left(\boldsymbol{x}^{\prime}\right)}{R^{3}}-3 \frac{\left(\boldsymbol{M}\left(\boldsymbol{x}^{\prime}\right) \cdot \boldsymbol{R}\right) \boldsymbol{R}}{R^{5}}\right]
\end{aligned}
$$

where $\Omega_{c}$ is the wire domain in which the magnetization $\boldsymbol{M}$ exists, $\boldsymbol{x}$ and $\boldsymbol{x}^{\prime}$ are the observation and source points in $\Omega_{c}, \boldsymbol{\tau}$ is the tangential unit vector parallel to the wire axis at $\boldsymbol{x}, \boldsymbol{R}=$ $\boldsymbol{x}-\boldsymbol{x}^{\prime}, R=|\boldsymbol{R}|$, and $G$ denotes the operator defined by (3b).

Then, we consider the stranded wires which have periodic structure in the direction of the wire axis as shown in Fig.1. Due to the periodic structure, the magnetization satisfies

$$
M(x+n d)=M(x)
$$

where $n$ denotes integer and $\boldsymbol{d}$ is the distance vector between the adjacent unit cells. The integral equation for the wire with periodic structure is, thus, can be expressed as

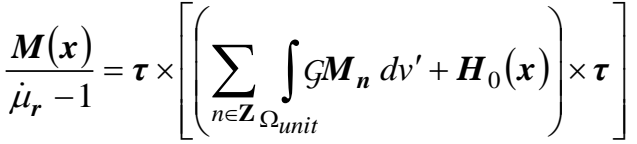

$$
\begin{aligned}
& G M_{n}=-\frac{1}{4 \pi}\left[\frac{\boldsymbol{M}\left(\boldsymbol{x}^{\prime}\right)}{R_{n}^{3}}-3 \frac{\left(\boldsymbol{M}\left(\boldsymbol{x}^{\prime}\right) \cdot \boldsymbol{R}_{n}\right) \boldsymbol{R}_{n}}{R_{n}^{5}}\right]
\end{aligned}
$$

where $\Omega_{\text {unit }}$ represents the repeating unit of $\Omega_{\mathrm{c}}, \boldsymbol{R}_{n}=\boldsymbol{x}-$ $\boldsymbol{x}^{\prime}-n \boldsymbol{d}, R_{n}=\left|\boldsymbol{R}_{n}\right|$. The summation in (5a) is terminated at adequate number considering both accuracy and computational time. In the following computations, we evaluate 11 terms.

\section{Macroscopic permeability}

To perform homogenization of a bundle of litz wires, we derive its macroscopic permeability from $\dot{\mu}_{r}$. To do so, we use $\boldsymbol{M}$ obtained by solving (5a) as will be described below.

Let the time-harmonic uniform magnetic field $\boldsymbol{H}_{0} e^{j \omega t}$, angular frequency $\omega$, be imposed on the unit cells as shown in Fig.1. We can obtain the distribution of $\boldsymbol{M}$ along the wires by solving the periodic integral equation (5a). The uniform magnetic field $\boldsymbol{H}_{0}$ in (5a) can be regarded as Lorentz field $\boldsymbol{H}_{\text {loc }}$ when we consider the unit cell which contains a bundle of stranded wire as shown in Fig.2. The following relation holds

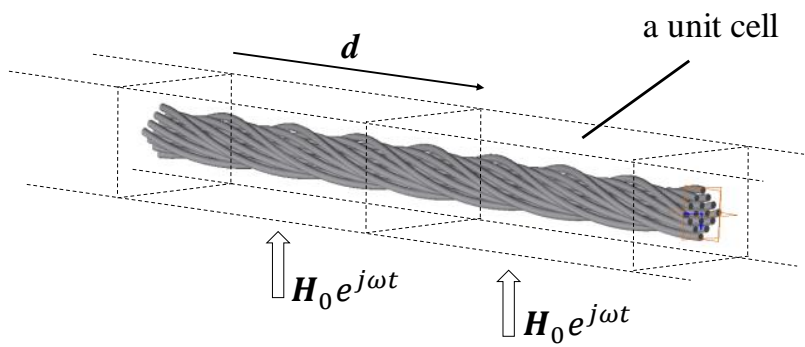

Fig.1. Litz wire immersed in time-harmonic magnetic field.

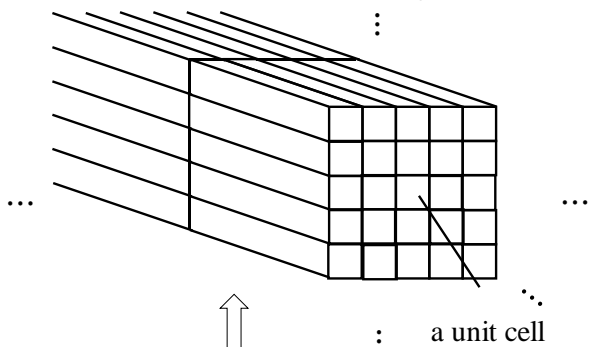

$$
\boldsymbol{H} e^{j \omega t}
$$

Fig.2. External magnetic field $\boldsymbol{H}$ is imposed to a bundle of litz wires. If we remove a unit cell, there left Lorentz field which is generated by $\boldsymbol{H}$ and the magnetization of the other unit cells.

for the Lorentz field:

$\boldsymbol{H}_{l o c}=\boldsymbol{H}+\bar{N} \boldsymbol{M}_{a v e}$

where $\boldsymbol{H}$ represents the external magnetic field, which is imposed on the entire cells, $\bar{N}$ denotes the effective diamagnetic constant of a unit cell, and $\boldsymbol{M}_{\text {ave }}$ is the averaged magnetization over a unit cell. The computation of $\bar{N}$ is given in Appendix A. Then the averaged magnetic flux density over the unit cell can be written as

$\boldsymbol{B}_{\text {ave }}=\mu_{0}\left(\boldsymbol{H}+\boldsymbol{M}_{\text {ave }}\right)$

On the other hand, by introducing the macroscopic permeability $\langle\dot{\mu}\rangle, \boldsymbol{B}_{\text {ave }}$ can be written as

$\boldsymbol{B}_{\text {ave }}=\left\langle\dot{\mu}_{\boldsymbol{r}}\right\rangle \mu_{0} \boldsymbol{M}_{\text {ave }}$

Eliminating $\boldsymbol{H}$ from (6), (7), and (8), we have

$$
\begin{aligned}
\left\langle\dot{\mu}_{\boldsymbol{r}}\right\rangle & =\frac{1}{1-\mu_{0} \frac{M_{\text {ave }}}{B_{\text {ave }}}} \\
\boldsymbol{B}_{\text {ave }} & =\mu_{0}\left(\boldsymbol{H}_{\text {loc }}-\bar{N} \boldsymbol{M}_{\text {ave }}+\boldsymbol{M}_{\text {ave }}\right)
\end{aligned}
$$

Note that this permeability is valid under the assumption that the magnetic field imposed on the litz wire is uniform.

\section{NUMERICAL RESULTS}

\section{A. Validation of the proposed method}

To validate the proposed method, a 3D model of a litz wire shown in Fig. 3 is analyzed by conventional FEM and the 
proposed method. The model is composed of 16 wires, wire radius $a=0.15 \mathrm{~mm}$, pitch $15 \mathrm{~mm}$, conductivity $\sigma=5.76 \times 10^{7}$ $\mathrm{S} / \mathrm{m}$, and relative permeability $\mu_{\mathrm{r}}=1.0$.

In the FE analysis, the uniform magnetic flux density is imposed to the unit cell. The boundary conditions are summarized in TABLE.1. The macroscopic permeability is computed from the following equation:

$$
\left\langle\dot{\mu}_{\boldsymbol{r}}\right\rangle=\frac{\boldsymbol{j} \omega \int_{\Omega_{\text {unit }}} \frac{\left|\boldsymbol{B}_{\text {ave }}\right|^{2}}{\mu} d v}{\boldsymbol{j} \omega \int_{\Omega_{\text {unit }}} \frac{|\boldsymbol{B}|^{2}}{\mu} d v+\int_{\Omega_{\text {unit }}} \sigma|\boldsymbol{E}|^{2} d v}
$$

where $\boldsymbol{B}_{\text {ave }}$ denotes the averaged magnetic flux density over the domain.

\begin{tabular}{cc} 
TABLE.1. BOUNDARY CONDITION OF FE MODEL \\
\hline \hline boundary planes & boundary condition \\
\hline$y z$ & Dirichlet \\
$x z$ & Neumann \\
$x y$ & Periodic \\
\hline \hline
\end{tabular}

The results are shown in Fig.4. The horizontal axis is the wire radius normalized to the skin depth $\delta$. We can see that $\langle\dot{\mu}\rangle$ obtained by the proposed method is in good agreement with that obtained by 3D FEM when $a<\delta$. The ratio of the computational time of the proposed method to that of FEM is about $1.4 \times 10^{-2}$ for one frequency sample. The proposed method is, therefore, more useful to evaluate the dependence of $\langle\dot{\mu}\rangle$ on number of wires, wire pitch and strand structure.

\section{B. Dependence of $\langle\dot{\mu}\rangle$ on pitch}

The dependence of $\langle\dot{\mu}\rangle$ on the pitch of a litz wire is evaluated from (9a). We consider a litz wire composed of 49 wires, radius $a=0.15 \mathrm{~mm}$, the conductivity $\sigma=5.76 \times 10^{7} \mathrm{~S} / \mathrm{m}$, relative permeability $\mu_{\mathrm{r}}=1.0$.

The imaginary part of $\langle\dot{\mu}\rangle$ is plotted against frequency in Fig.4. It is found that $\langle\dot{\mu}\rangle$ scarcely depends on the pitch under the condition that $a<\delta$ which holds for usual litz wires. In Fig.5, $\langle\dot{\mu}\rangle$ computed from Ollendorff formula [5][9]

$$
\left\langle\dot{\mu}_{r}\right\rangle=1+\frac{2 \eta\left(\dot{\mu}_{r}-1\right)}{2+(\eta-1)\left(\dot{\mu}_{r}-1\right)}
$$

in which wires are assumed to be in parallel is also plotted, where $\eta$ represents the filling ratio. From this result, we conclude that the proximity effect losses can be efficiently evaluated from (11) for the litz wire shown in Fig.3. Namely, the wire twist has little effect on $\left\langle\dot{\mu}_{r}\right\rangle$ when $a<\delta$.

\section{EXPERIMENTAL RESULT}

We consider the AC resistance of the wireless power transfer (WPT) coil composed of the litz wire shown in Fig.6(a). The parameters are summarized in TABLE.2. The AC resistances computed by FEM and measured values are plotted against frequency in Fig.6(b). The macroscopic permeability $\langle\dot{\mu}\rangle$ obtained from (9a) is used in the FE analysis. We can see

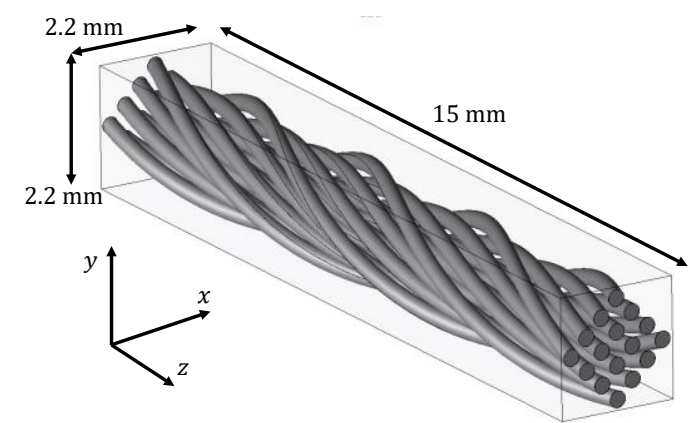

Fig.3. A unit cell of litz wire model composed of 16 wires.

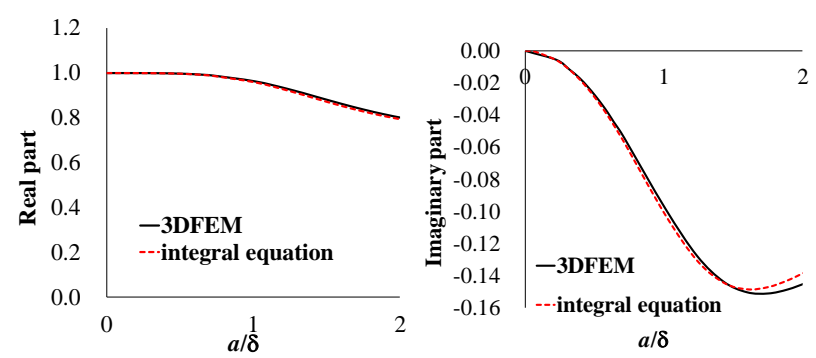

Fig.4. Real (left) and imaginary (right) parts of macroscopic permeability $\langle\dot{\mu}\rangle$ are plotted against the normalized wire radius.

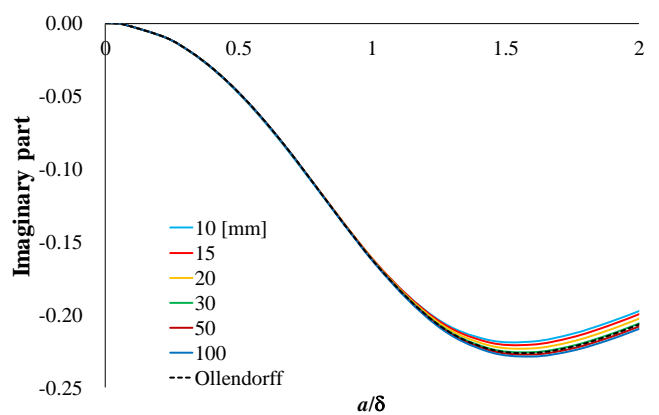

Fig.5. Imaginary part of macroscopic permeability of 49 stranded wires is plotted against the normalized wire radius.

TABLE.2. PARAMETERS OF WPT COIL

\begin{tabular}{cc} 
Strand number & 50 \\
Radius [mm] & 0.025 \\
Turn number & 40 \\
nner diameter [mm] & 30 \\
Outer diameter [mm] & 74 \\
Thickness [mm] & 0.8 \\
\hline
\end{tabular}

the computed AC resistance agrees well with the measured values except at high frequencies where the capacitance among the wires would not be negligible.

\section{DISCUSSION}

In this paper, we have discussed the eddy currents losses due to the proximity effect. We find that wire twist has little effects on $\left\langle\dot{\mu}_{r}\right\rangle$ whose imaginary part represents the AC loss due the proximity effect when $a<\delta$. However, there exists other factors that can cause AC losses in the wires. The main factors are the skin effect and circulation currents. Here, we discuss the latter because the former can be sufficiently reduced when using litz wires. The latter effect has not been included in the analysis of eddy current losses of litz wire [10-12].

The circulation currents are the currents which flow along 
two wire conductors electrically connected at their terminals. For example, we consider the twisted wires shown in Fig.7. When magnetic flux interlinkages across the wires, the circulation currents flow along the wires due to Faraday's law. If the wires are ideally well twisted and the magnetic field is uniform, the circulation currents cancel out each other. In practice, however, the wires are not always ideally twisted and the magnetic field can vary along the wires. The circulation currents, therefore, can contribute to the AC losses. In [13], the current sharing has been determined by solving the circuit equation resulted from 2.5D PEEC method. This method leads to, however, a huge equation system when the litz wire contains a number of strands.

The AC losses due to the circulation currents could be evaluated in the post-processing; the wire is modeled as a curve or segmented lines and the eddy currents along them are computed from the interlinkage flux. This remains for future work.

\section{CONCLUSION}

In this paper, we have proposed a new method to obtain the macroscopic permeability $\langle\dot{\mu}\rangle$ of a litz wire using integral equation method. By using this method, we can evaluate $\langle\dot{\mu}\rangle$ without heavy computational burden. It has been shown that the macroscopic permeability of the litz wire obtained by the proposed method is in good agreement with that obtained by the Ollendorff formula. Thus, it is concluded that the eddy current losses due to the proximity effect can be accurately evaluated by the Ollendorff formula. This result has also been verified by experiment. We have also discussed the circulation current which gives rise to additional AC losses. The evaluation of the circulation current losses in the litz wire is our future work.

\section{ACKNOWLEDGEMENT}

This work was supported in part by KAKENHI $15 \mathrm{H} 02976$.

\section{APPENDIX A}

We consider here the effective diamagnetic constant of a stranded wire. By definition, it would be expressed as

$\bar{N}=\frac{H_{0}-H_{a v e}^{i n}}{M_{a v e}^{i n}}$

where $\boldsymbol{H}_{\text {ave }}^{i n}$ is the averaged magnetic field over the stranded wires. The averaged magnetic field is computed from

$$
\boldsymbol{H}_{\text {ave }}^{\text {in }}=\frac{\boldsymbol{M}_{\text {ave }}^{\text {in }}}{\mu_{r}-1}
$$

where $\boldsymbol{M}_{\text {ave }}^{\text {in }}$ is the magnetization, computed by solving (5a) with real-valued permeability, averaged over the conductor.

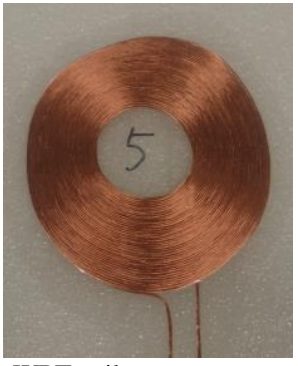

(a) WPT coil

Fig.6. WPT coil and its AC resistance.

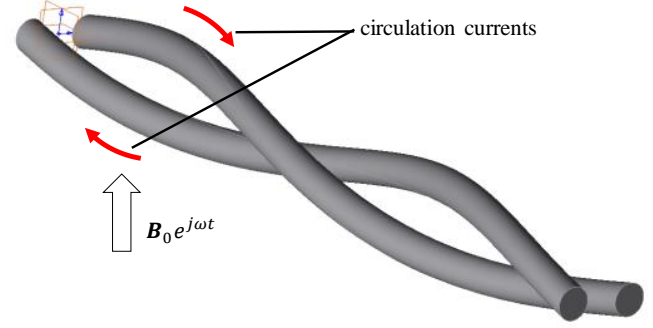

Fig.7. Circulation currents in a pair of wires.

\section{REFERENCES}

[1] A. D. Podoltsev, I. N. Kucheryavaya, B. B. Lebedev, "Analysis of Effective Resistance and Eddy-Current Losses in Multiturn Winding of High-Frequency Mangetic Components," IEEE Trans. Magn., vol.39, no.1, January 2003.

[2] Xi Nan, C. Sullivan, "An Equivalent Complex Permeability Model for Litz-wire windings," in IEEE Ind. Appl. Soc. Ann. Meeting, 2005, pp.2229-2235.

[3] J. Gyselinck, P. Dular, "Frequency-Domain Homogenization of Bundles of Wires in 2-D Magnetodynamic FE Calculations," IEEE Trans. Magn., vol. 41, no. 5, pp. 1416-1419, May, 2005.

[4] H. Waki, H. Igarashi, T. Honma, "Estimation of Effective Permeability of Magnetic Composite Materials Magnetics," IEEE Trans.Magn., vol. 41, no. 5, pp. $1520-1523,2005$.

[5] H. Igarashi, "Semi-Analytical Approach for Finite Element Analysis of Multi-turn Coil Considering Skin and Proximity Effects," IEEE Trans. Magn., vol.53, no.1, January 2017.

[6] Y. Sato, H. Igarashi, "Homogenization Method Based on Model Order Reduction for FE Analysis of Multi-turn Coils," IEEE Trans. Magn., vol.53, no.6, June 2017.

[7] Y. Sato, H. Igarashi, "Time-domain Analysis of Soft Magnetic Composite Using Equivalent Circuit Obtained via Homogenization," IEEE Trans. Magn., vol.53, no.6, June 2017.

[8] S. Hiruma, H. Igarashi, "Fast Three-Dimensional Analysis of Eddy Current in litz Wire Using Integral Equation," IEEE Trans. Magn., vol.53, no.6, June 2017.

[9] F. Ollendorff, "Magnetostatik der Massekerne," Arch.f. Elektrotechnik., 25, pp. 436-447, 1931.

[10] Q. Deng, J. Liu, D. Czarkowski, M. K. Kazimierczuk, M. Bojarski, H. Zhou, W. Hu, "Frequency-Dependent resistance of Litz-Wire Square Solenoid Coils and Quality Factor Optimization fo Wireless Power Transfer," IEEE Trans. Ind. Elec., vol. 63, no. 5, May 2016.

[11] D. C. Meeker, "An improved Continuum Skin and Proximity Effect Model for Hexagonally Packed Wires," Journal of Computational and Applied Mathematics, Volume 236, Issue 18, 2012, Pages 4635-4644, ISSN 0377-0427, http://dx.doi.org/10.1016/j.cam.2012.04.009.

[12] C. R. Sullivan, R. Y. Zhang, "Analytical Model for Effects of Twisting on Litz-Wire Losses," Control and Modeling for Power Electronics (COMPEL), 2014 IEEE 15th Workshop on. IEEE, 2014.

[13] T. Guillod, J. Huber, F. Krismer J. W. Kolar, Litz Wire Losses: Effects of Twisting Imperfections, Proceedings of the 18th IEEE Workshop on Control and Modeling for Power Electronics (COMPEL 2017), Stanford, California, USA, July 9-12, 2017. 